\title{
LEAD V4R IN RIGHT VENTRICULAR HYPERTROPHY
}

\author{
BY \\ F. CAMERINI*, J. F. GOODWIN, AND M. ZOOB \\ From the Department of Medicine, Postgraduate Medical School of London
}

Received March 30, 1955

The cardiographic criteria generally used for the diagnosis of right ventricular hypertrophy are those proposed by Myers et al. (1948) following the work of Wilson et al. (1944). Experience of these criteria has shown that although they rarely give false positive results, many cases of right ventricular hypertrophy are missed. To meet this difficulty, Myers and his associates suggested the use of leads from positions further to the right of the sternum, and mentioned two instances in which a diagnosis of right ventricular hypertrophy was made from the appearance of V3R when the diagnostic pattern was absent from the remaining leads. Rosenman et al. (1950) also found V4R essential for the diagnosis of right ventricular hypertrophy in 5 of 25 cases but no systematic evaluation of this lead has been made.

In the present study the pattern of V4R has been examined in a control series of autopsies, in a series with pathological proof of right ventricular hypertrophy, and in a clinical series of adults with evidence of right ventricular hypertrophy. In addition we have compared the relative value of V4R, V1, and aVR.

Pathological Methods and Criteria. Assessment of the degree of ventricular hypertrophy at autopsy is difficult, but an attempt was made by taking the measurement of maximum thickness of the ventricle in the region of the outflow tract, and by employing the classification of Pagnoni and Goodwin (1952). Right ventricular thickness between 5 and $7 \mathrm{~mm}$. was described as slight, $8-10 \mathrm{~mm}$. as moderate, and $10 \mathrm{~mm}$. or more as severe. Left ventricular thickness between 15 and $18 \mathrm{~mm}$. was described as slight, 19-24 mm. as moderate, and $24 \mathrm{~mm}$. or more as severe. Cases with cardiac infarction were excluded.

Cardiographic Methods and Criteria. V4R was recorded from the right chest anteriorly at the intersection of the mid-clavicular line with the fifth intercostal space. In all patients, the standard and unipolar limb leads, and V1, V2, V4, and V6 were recorded. In some instances V5R, V3R, V3, V5, and V7 were also obtained. The criteria used for the diagnosis of right ventricular hypertrophy were those of Myers et al. (1948).

The term incomplete right bundle-branch block has been avoided. Instead, the description $\mathrm{rSr}^{\prime}$ has been applied to QRS complexes of a duration of less than $0 \cdot 12 \mathrm{sec}$. showing either secondary $r^{\prime}$ wave smaller than the $S$ wave, or an embryonic $r^{\prime}$ wave notching the $S$ wave; the term $\mathrm{rSR}^{\prime}$ has been applied to complexes in which the secondary $R$ wave was greater than both the initial $r$ wave and the $s$ wave.

Material. The study is divided into three parts.

I. Controls. The electrocardiograms of 73 subjects in whom autopsy showed no right ventricular hypertrophy were studied. There were 25 cases of left ventricular hypertrophy ranging in degree from slight to severe, and in the remainder, no ventricular hypertrophy was present. The age of these cases ranged from 18 to 88 years.

II. Electrocardiograms were studied of 31 cases in which autopsy showed hypertrophy of the right ventricle alone, and in 52 cases in which hypertrophy of both ventricles was present.

* During the tenure of a World Health Organization Fellowship. 
III. A series of a further 96 electrocardiograms showing abnormalities in V4R and/or in V1 was studied and comparison made of the frequency of occurrence of abnormalities of the two leads.

\section{RESULTS}

I. Controls. In 64 of the 73 autopsy cases in which the right ventricle was normal, V4R showed $\mathrm{rS}$ or QS patterns resembling those normally found in V1. The $\mathrm{rSr}^{\prime}$ pattern was found in 4 cases, $\mathrm{rSR}^{\prime}$ in one, and there was one example each of left and right bundle-branch block. In two instances a $Q$ wave was present without evidence of infarction, and in both of these it seemed that an isoelectric beginning of the QRS may have caused complexes which were really $\mathrm{rSr}^{\prime} \mathrm{s}^{\prime}$ and $\mathrm{rSR}^{\prime}$ to simulate QRS and QR complexes respectively.

Throughout this control group the $R$ wave was smaller than the $S$ wave in V4R as well as in V1. It was therefore concluded that the pattern $R>S$ in V4R could be tentatively regarded as diagnostic of right ventricular hypertrophy provided that children and patients with ischæmic heart disease were excluded.

On the other hand $\mathrm{rSr}^{\prime}$ complexes have been shown to occur commonly in right præcordial leads in normal subjects (Camerini and Davies, 1955), and although a secondary $\mathbf{r}^{\prime}$ wave tends to grow larger as right-sided hypertrophy develops (Mounsey et al., 1952a), rSR' complexes may nevertheless occur in its absence. One such case was found in this control group, in addition to one example of complete right bundle-branch block. These patterns can therefore be regarded only as suggestive signs, and in the succeeding parts of this study, only the pattern $R>S$ in V4R or V1 has been regarded as diagnostic of right ventricular hypertrophy.

II. (a) Lone Right Ventricular Hypertrophy. Of the 31 cases with lone ventricular hypertrophy at autopsy, the $R>S$ pattern was present in V4R in 11 and in $V 1$ in only 4 of these. In the seven cases in which the $R>S$ pattern was found only in V4R, V1 showed normal patterns in 5 and rSR' complexes in two. Lead aVR showed a dominant $R$ wave in four cases (Fig. 1).

The finding in the 20 cases in which neither V4R nor V1 were diagnostic were as follows:

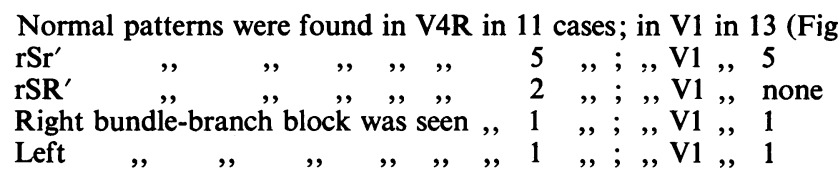

In these 20 cases, hypertrophy was slight in 12 and moderate in 6: in one of the two severe cases right bundle-branch block was present; in the other, both V4R and V1 were normal.

(b) Combined Ventricular Hypertrophy. The R $>\mathrm{S}$ pattern was found in V4R in 11 of the 52 patients $(21 \%)$ and in V1 in only 6 of these $(12 \%)$. In the other 5 cases in which V4R was diagnostic $\mathrm{V} 1$ showed normal patterns in 3 and $\mathrm{rSr}^{\prime}$ patterns in 2 (Fig. 3). Lead aVR showed a dominant $R$ wave in 6 cases (Fig. 4).

The findings in the 41 cases in which neither V4R nor V1 were diagnostic of right ventricular hypertrophy were as follows.

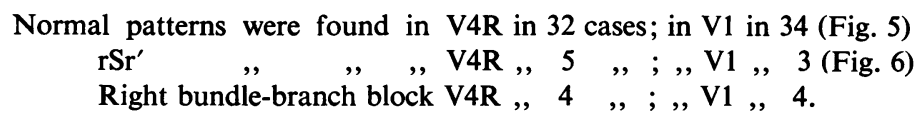

Of these 41 cases, 31 had moderate or severe left ventricular hypertrophy. Of the remaining 10 with slight left-sided hypertrophy, the right ventricular hypertrophy was slight in 6 , and moderate in 4 cases.

III. The 96 patients whose electrocardiograms showed abnormalities in V4R and/or in V1 have been considered in three groups according to the pattern found: (i) 66 patients showed an $R>S$ pattern, (ii) 20 patients showed an $\mathrm{rSr}^{\prime}$ complex, and (iii) 10 patients showed an rSR' pattern.

(i). In this group only 27 patients showed a dominant $R$ wave in aVR, and 28 an $R>S$ in V1. 


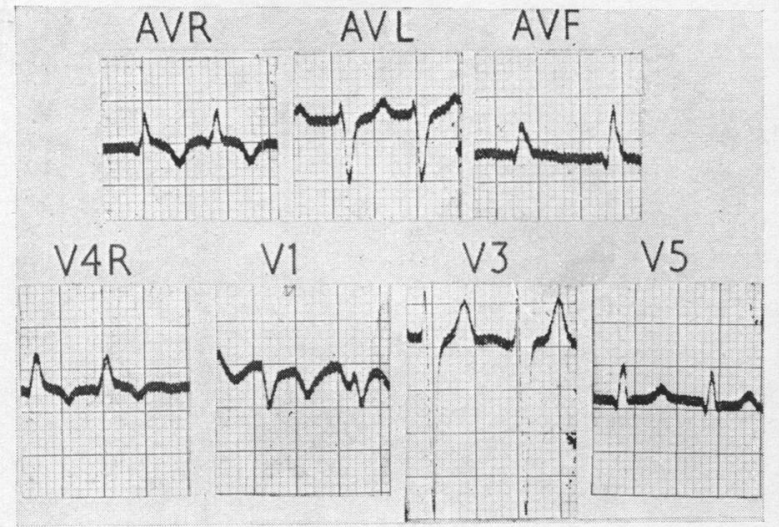

FIG. 1.-Cardiogram of patient with autopsy evidence of severe lone right ventricular hypertrophy. A dominant $R$ wave is present in aVR and V4R but not in V1. Rhythm, auricular flutter.

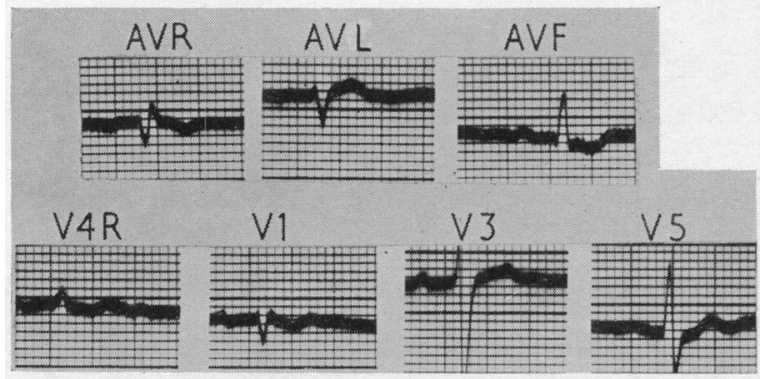

FIG. 3.-Cardiogram of patient with autopsy evidence of moderate hypertrophy of the right ventricle, and slight hypertrophy of the left ventricle. V4R shows a small $R$ wave, while $V 1$ shows $\mathrm{rSr}^{\prime}$. There is a Qr pattern in aVR.

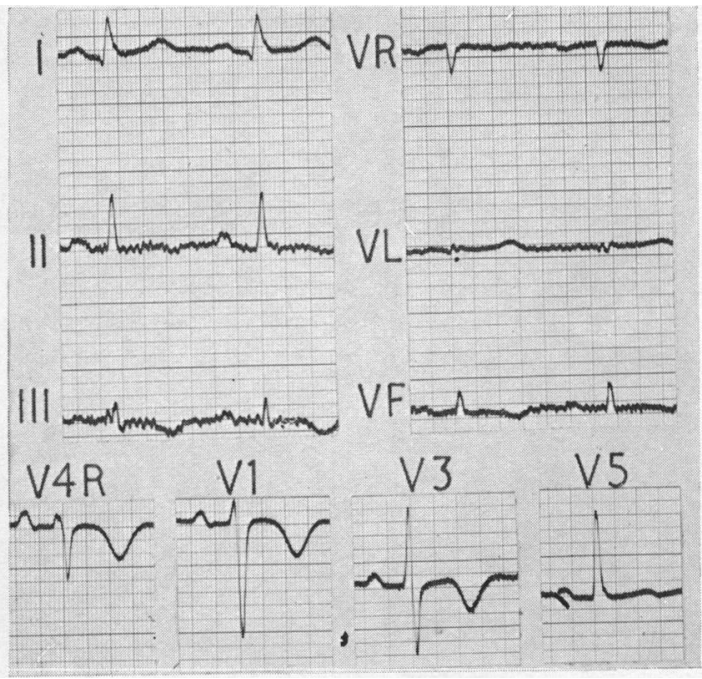

FIG. 2.-Cardiogram of patient with autopsy evidence of slight lone right ventricular hypertrophy. Leads V4R, V1, and aVR, show rS patterns. $T$ wave inversion in V4R, V1, and V3 alone suggests right ventricular strain.

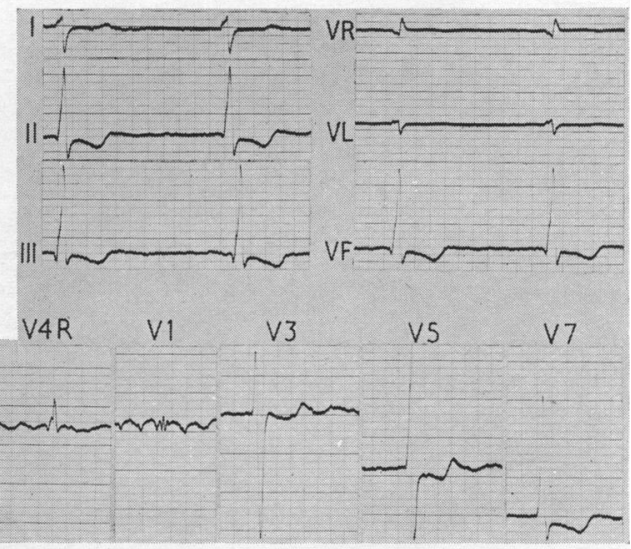

FIG. 4.-Cardiogram of patient with autopsy evidence of moderate right and left ventricular hypertrophy. V4R shows a dominant $R$ wave and $\mathrm{V} 1$ an $\mathrm{rSr}^{\prime}$. aVR shows a qR pattern. Rhythm, auricular fibrillation.

Only one patient showed $\mathrm{R}>\mathrm{S}$ in V1 when it was not present in V4R. By contrast, 38 patients showed $\mathrm{R}>\mathrm{S}$ in V4R alone; in these cases V1 showed $\mathrm{rS}$ or QS in 17 (Fig. 7), $\mathrm{rSr}^{\prime}$ in $\lambda 10$ (Fig. 8), $\mathrm{rSR}^{\prime}$ in 8, and QRS patterns in 3. In these patients, the conclusive diagnosis of right ventricular hypertrophy depended on V4R alone, and in 30 of them, there was clinical or radiological evidence supporting this diagnosis: of the remainder, right ventricular hypertrophy might have beeı expected in 2, but was unexpected in 6 cases who presented no corroborative evidence of it. Of the 27 patients who showed $R>S$ in both V4R and V1, 26 had supporting clinical and radiological evidence of right-sided hypertrophy, but in the remaining case the finding was unexpected. 


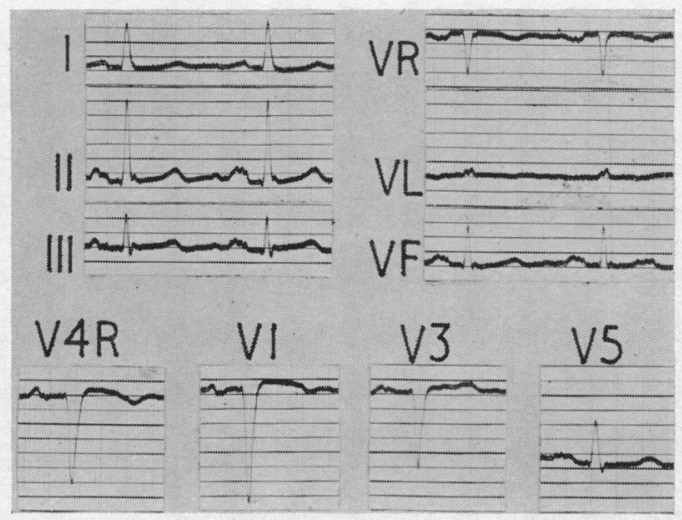

FIG. 5.-Cardiogram of patient with autopsy evidence of moderate hypertrophy of both ventricles. Leads V4R, V1 and aVR all show normal patterns: no evidence of left or right ventricular hypertrophy.

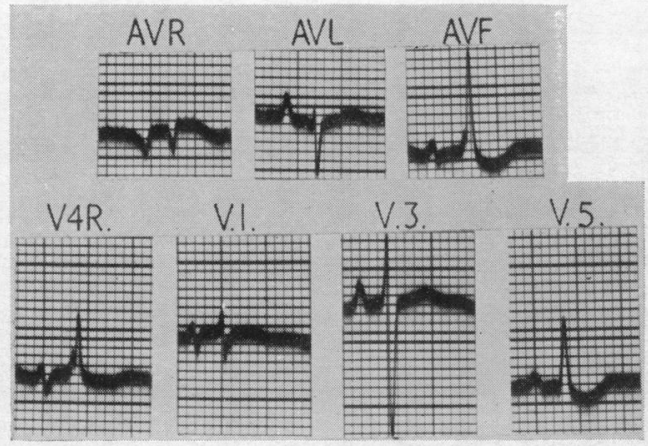

Fig. 7.-Cardiogram of patient with chronic cor pulmonale and clinical evidence of right ventricular hypertrophy. Lead V4R shows a dominant $R$ wave and V1 an rS pattern. aVR shows a Qr complex. V4R is thus the sole sign of right ventricular hypertrophy.

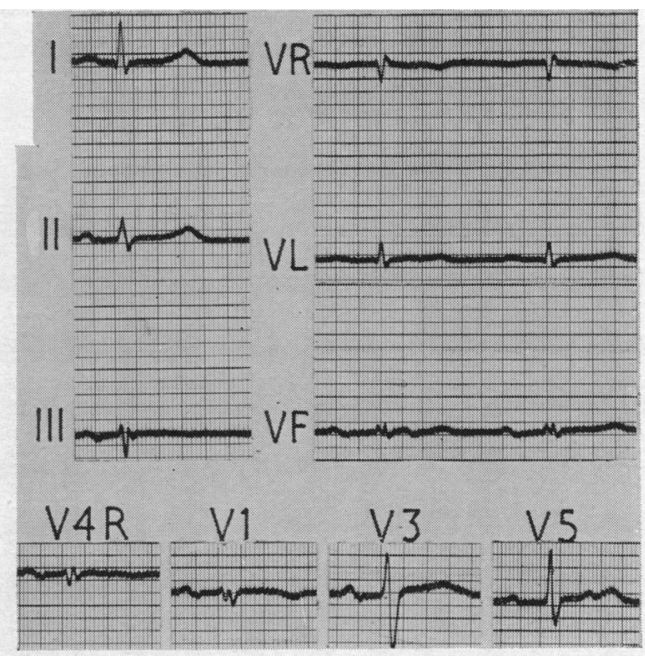

FIG. 6.-Cardiogram of patient with autopsy evidence of moderate right ventricular hypertrophy, and slight left ventricular hypertrophy. Leads V4R, V1, and aVR all show rsr' patterns.

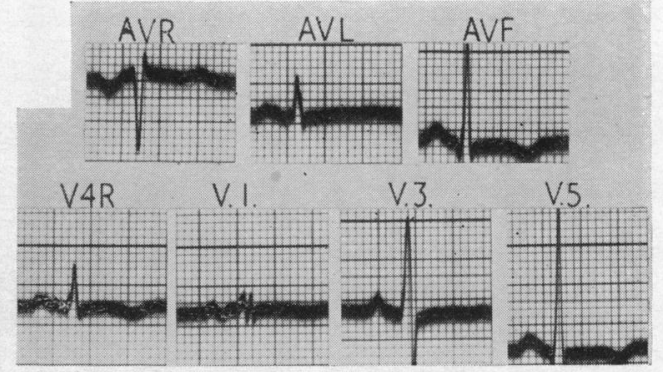

Fig. 8.-Cardiogram of patient with mitral stenosis and clinical evidence of right ventricular hypertrophy. V4R shows a dominant $R$ wave, and $a V R$, and $V 1$ $\mathrm{rSr}^{\prime}$ patterns.

(ii). Of the 20 patients in this group, 9 showed $\mathrm{rSr}^{\prime}$ patterns in $\mathrm{V} 4 \mathrm{R}$ together with normal patterns in $\mathrm{V} 1$; the remainder showed $\mathrm{rSr}^{\prime}$ patterns in both leads. Four patients showed a dominant $\mathrm{R}$ wave in aVR. In two of the patients in whom $\mathrm{rSr}^{\prime}$ was present in $\mathrm{V} 4 \mathrm{R}$ alone, and in two in whom it was present in both leads, the clinical evidence was not suggestive of right ventricular hypertrophy. In the remainder, the clinical findings were compatible with that diagnosis.

(iii). Of the 10 patients in whom V4R showed $\mathrm{rSR}^{\prime}$ complexes, aVR showed a dominant $\mathrm{R}$ wave in $1, \mathrm{~V} 1$ showed $\mathrm{rSR}^{\prime}$ patterns in $6, \mathrm{R}>\mathrm{S}$ in 1 , and $\mathrm{rSr}^{\prime}$ in 3 . In one of the last, the clinical findings were not suggestive of right ventricular hypertrophy; in the remainder they were suggestive or at least compatible with that diagnosis. 
Discussion

It is probable that the pattern $R>S$ in V4R in adult subjects is almost invariably diagnostic of right ventricular hypertrophy. In our autopsy series, confirmation of a diagnosis based on this sign was always obtained and the pattern was found most frequently among cases with marked hypertrophy. The diagnostic appearance was not seen in the control group in which right ventricular hypertrophy was absent. Cases of cardiac aneurysm and postero-lateral infarction must, however, be excluded from this generalization, for Levy et al. (1950) have shown that these conditions may produce a dominant $\mathrm{R}$ wave in $\mathrm{V} 1$ and we have also found it in V4R in such cases. Also the pattern may occur in V4R and V1 transiently in the course of acute or chronic bronchitis or temporary right ventricular strain (Fig. 9 and 10). The pulmonary artery pressure is known to be transiently raised in such circumstances (Mounsey et al. $(1952 b)$, but it seems unlikely that hypertrophy develops and disappears within a few days. The pattern in these cases, as indeed in those associated with hypertrophy, may be due to alteration in the position of the heart. This will be further discussed. There remain six cases in which an $R>S$ pattern in V4R was the only sign of right ventricular hypertrophy of which there was no corroborative evidence elsewhere in the cardiogram or in the clinical findings. It would seem that in such circumstances caution must be exercised before undue significance is attached to a single cardiographic sign.

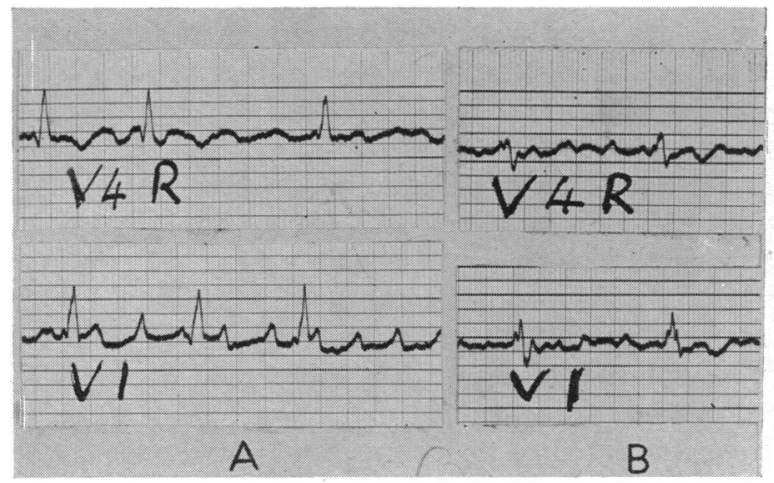

FIG. 9.-(A) Leads V4R and V1 from the cardiogram of a patient with mitral stenosis and auricular fibrillation taken during an episode of increased right ventricular stress. Both leads show unequivocally dominant $\mathbf{R}$ waves indicating right ventricular enlargement. (B) The same leads from the same patient after clinical improvement. There is now a definite $S$ wave, and the $R$ wave is smaller, suggesting less marked right ventricular stress, although there is some variation in the complexes from beat to beat.

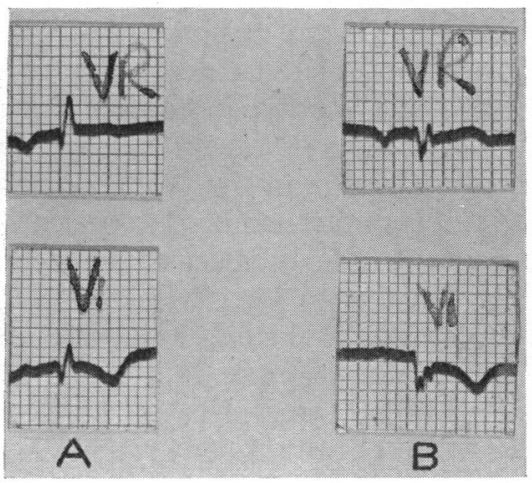

FIG. 10.-(A) Leads aVR and V1 from the cardiogram of a patient with cor pulmonale and an exacerbation of bronchitis. Both leads show dominant $\mathbf{R}$ waves indicating right ventricular enlargement. (B) The same leads from the same patient ten days later, after resolution of the acute attack. There is a predominantly negative deflection in both leads, suggesting resolution of the acute right ventricular stress.

The significance of $\mathrm{rSR}^{\prime}$ patterns is less clear cut. They may occur as a stage in the evolution of right bundle-branch block, which itself is not invariably associated with right ventricular hypertrophy. On the other hand they were not found in normal subjects by Camerini and Davies (1955), and occurred only once in our control series of autopsies without right ventricular hypertrophy. In the others in which this pattern was seen, right ventricular hypertrophy was proven at autopsy in 2 , and was clinically suspect in 9 , but probably absent in 1 case. It follows that this pattern is suggestive but not diagnostic of right ventricular hypertrophy. It was found in 12 cases in V4R and in 16 cases in V1. In 4 cases V4R showed $R>S$ when V1 showed rSR'.

The $\mathrm{rSr}^{\prime}$ pattern occurs commonly in right præcordial leads in normal subjects (Camerini and C 
Davies, 1955). On the other hand, Mounsey et al. (1952a) have shown that an "embryonic " $\mathrm{r}^{\prime}$ wave may appear and grow progressively larger as right ventricular hypertrophy develops in the course of pulmonary heart disease. It follows, therefore, that a single record showing this pattern is in no way diagnostic of right ventricular hypertrophy, but its appearance or progression is suggestive. In all cases in our series in which V1 showed this pattern, V4R showed either the same pattern or one more strongly suggestive of right ventricular hypertrophy.

The accuracy of the cardiographic diagnosis of right ventricular hypertrophy was considerably improved by the use of lead V4R which showed a diagnostic pattern in 27 per cent of autopsyproved right ventricular cases, compared with 12 per cent in V1 and aVR. In the remaining cases suggestive $\mathrm{rSr}^{\prime}$ and $\mathrm{rSR}^{\prime}$ patterns were found as often in V4R as in V1. The frequency with which even V4R was diagnostic, was nevertheless low. This was partly accounted for by the inclusion of cases of combined ventricular hypertrophy. In the latter group, the greater the degree of left ventricular hypertrophy, the less frequently was the $R>S$ pattern seen in V4R. In cases of lone right ventricular hypertrophy, V4R was diagnostic in 35 per cent and failures occurred most often in the cases with slight hypertrophy.

Taking the clinical and autopsy series together, there were 81 cases of proved or probable right ventricular hypertrophy in which $R>S$ was seen in V4R and/or V1. In $44(54 \%)$ it was found in V4R alone but in only one instance was it present in V1 alone, V4R showing rSR'.

The mode of production of the $\mathrm{R}>\mathrm{S}$ pattern in V1 has been the subject of much controversy. Those workers who assume that præcordial lead patterns are derived predominantly from the activation of the muscle underlying the electrode have naturally supposed that $\mathrm{qR}$ or Rs patterns represented the specific pattern of the hypertrophic right ventricle. The presence of an initial negative deflection ( $Q$ wave) has been attributed by Myers (1950) to one of two factors: either abnormal activation of the septum or normal activation but with the production of so small an $r$ wave that it is not transmitted to præcordial leads. Fowler et al. (1950) found that initial negativity in the right ventricular cavity could occur in subjects with right ventricular hypertrophy and thought that this was due to interference with the normal left-to-right septal depolarization. Possibly in some cases the initial small $r$ waves due to normal septal activation are not recorded in V1 owing to spread of the impulse in a plane parallel with that of the lead. This would cause the beginning of the QRS interval to be iso-electric; the initial downward deflection, which by definition must be called $\mathrm{Q}$, would be in reality an $\mathrm{S}$ wave and the final deflection would really be a secondary $\mathrm{R}$ wave. The whole complex would then be essentially an $\mathrm{rSR}^{\prime}$ pattern, of which the final $\mathrm{R}^{\prime}$ might represent activation of the right ventricle.

However, McGregor (1950) and Carouso et al. (1952) failed to find qR patterns in direct leads obtained from the right ventricular surface at operation, and McGregor (1950) and Sodi-Pallares et al. (1952) have suggested that such patterns are produced by rotation of the heart.

The assumption that præcordial lead patterns are derived predominantly from the muscle underlying the electrode has recently been subjected to much criticism by Scherlis et al. (1951), v. Luterotti (1951), Milnor et al. (1953), and Grishman et al. (1953). This assumption would appear to be wrongly based on the work of Wilson et al. (1944), who, although emphasizing the similarity between direct and præcordial leads, nevertheless stated ..." that, although the excitation of the muscle in contact with the exploring electrode produces a much larger and much more sudden fluctuation in the potential of this electrode, than the excitation of any equal mass of muscle at a greater distance from it, every unit of ventricular muscle, without exception, produces action currents which contribute to the form of these complexes."

The results of our study strongly suggest that $\mathrm{qR}$ and Rs patterns are not predominantly derived from the potential variations of the right ventricle. The magnitude of potential variations recorded by a remote electrode varies inversely with the cube of its distance from the electrical source. It follows that the more remotely an electrode is placed from the heart, the more equally it is influenced by the potential variations of the two ventricles. If $\mathrm{qR}$ or $\mathrm{Rs}$ patterns were predominantly derived from the right ventricle they would be expected to be recorded most frequently and conspicuously 
from the position closest to the right ventricle. In fact the reverse was found to be the case; V4R showed the $\mathrm{qR}$ and RS pattern more frequently and more conspicuously than V1. Since V4R is a remote lead and may be influenced fairly equally by the potential variations of all parts of the heart, it is probable that the abnormal $\mathrm{R}>\mathrm{S}$ pattern displayed by it is a manifestation of the alteration in the direction of the vector loop in the horizontal plane, which has been shown to occur in right ventricular hypertrophy (Lasser et al., 1951; Grishman and Scherlis, 1953; Shillingford and Brigden, 1954). This alteration is probably due to a combination of factors, including alteration of the relative position of the two ventricles, the relative magnitude of their electrical forces, and the direction of spread of the impulse. Altered direction of the horizontal vector loop may also account for $\mathrm{qR}$ and RS patterns in V1. It seems to us most unlikely that V1 can ever be regarded as a semidirect lead from the right ventricle. In normal subjects the fourth intercostal space at the right sternal border overlies the right atrium (Gray's Anatomy, 1954), and cardiac catheterization has shown that the tricuspid valve is a mid-line structure and may be displaced to the left in right ventricular hypertrophy. It follows that both in normal subjects and cases of right-sided hypertrophy, lead V1 overlies the right atrium and records the sum of the potential variations of that chamber together with those of the rest of the heart. There would therefore appear to be no grounds for the widespread assumption that $\mathrm{qR}$ and RS patterns recorded from V1 represent the potential variations of the epicardial surface of the right ventricle.

The view that both V4R and V1 should be regarded as remote leads, is supported by the fact that aVR, which cannot be supposed to be dominated by right ventricular potentials, frequently shows $\mathrm{qR}$ or RS patterns resembling those found in the same case in V4R and V1, and such patterns have been shown to be reliable evidence of right ventricular hypertrophy (Goodwin, 1952).

\section{SUMMARY}

The cardiograms of 252 adult subjects were studied with special reference to leads V4R, V1, and aVR. Cases of ischæmic heart disease were excluded. An rS pattern was the usual finding in V4R and V1 in a control series of 73 cases with no autopsy evidence of right ventricular hypertrophy. Although an rsr' occurred twice, and an rSR' once, an Rs pattern was not seen.

Eleven of 31 cases with autopsy evidence of right ventricular hypertrophy showed a dominant $\mathbf{R}$ wave diagnostic of this in V4R, but it was present also in V1 in only 4. Normal rS, rsr', or rSR' patterns occurred in the others. In a series of 52 cases with autopsy evidence of combined ventricular hypertrophy, the Rs pattern was present in V4R in only 11, and in V1 in 6 of these.

The cardiograms of 96 patients with clinical evidence suggesting right ventricular hypertrophy were also examined: 66 patients showed an Rs pattern in V4R, but less than half of these also had this pattern in V1 or aVR. Only one had an Rs pattern in V1, when it was not present in V4R.

The results indicate that V4R is a valuable lead in the diagnosis of right ventricular hypertrophy, often presenting a diagnostic Rs pattern in cases in which this is not present in V1 or aVR. Despite this, a proportion of cases remains in which V4R is normal. The Rs pattern was seen in V4R most frequently in subjects with considerable, rather than slight, right ventricular hypertrophy.

It is concluded that an $\mathrm{rSr}^{\prime}$ pattern in $\mathrm{V} 4 \mathrm{R}$ or $\mathrm{V} 1$ alone is not diagnostic of right ventricular hypertrophy, while an rSR' is suggestive, but not diagnostic. The genesis of the diagnostic Rs pattern in V4R and V1 is discussed, and it is suggested that these are remote rather than semidirect leads.

\section{REFERENCES}

Camerini, F., and Davies, L. G. (1955). Brit. Heart J., 17, 28.

Carouso, G. J., Chevalier, H. A., Latscha, I., and Lenègre, J. (1952), Circulation, 5, 48.

Fowler, N. O., Westcott, R. N., and Scott, R. C. (1952). Circulation, 5, 441.

Goodwin, J. F. (1952). Brit. Heart J., 14, 173.

Gray's Anatomy, (1954), 31 st ed., Longmans Green \& Co., London, p. 705.

Grishman, A., Scherlis, L., and Lasser, R. P. (1953). Amer. J. Med., 14, 184.

Lasser, R. P., Borun, E. R., and Grishman, A. (1951). Amer. Heart J., 42, 370. 
Levy, L., Jacobs, H. J., Chastant, H. P., and Strauss, H. B. (1950). Amer. Heart J., 40, 447. v. Lutterotti, H. (1951). Z. Kreislaufforsch, 40, 672.

McGregor, M. (1950). Brit. Heart J., 12, 351.

Milnor, W. R., Talbot, S. A., and Newman, E. V. (1953). Circulation, 7, 545.

Mounsey, J. P. D., Ritzmann, L. W., and Selverstone, N. J. (1952a). Brit. Heart J., 14, 442.

,,--- , and Briscoe, W. A. (1952b). Brit. Heart J., 114, 153.

Myers, G. B. (1950). Circulation, 1, 860.

$\longrightarrow$, Klein, H. A., and Stofer, B. E. (1948). Amer. Heart J., 35, 1.

Pagnoni, A., and Goodwin, J. F. (1952). Brit. Heart J., 14, 451.

Rosenman, R. H. (1950). Amer. Heart J., 40, 522.

Scherlis, L., Lasser, R. P., and Grishman, A. (1951). Amer. Heart J., 42, 235.

Shillingford, J., and Brigden, W. (1954). Brit. Heart J., 16, 13.

Sodi-Pallares, D., Bisteni, A., and Herrmann, G. R. (1952), Amer. Heart J., 43, 716.

Wilson, F. N., Johnston, F. D., Rosenbaum, F. F., Erlanger, H., Kossmann, C. E., Hecht, H., Cotrim, N., Menezes de Oliveira, R., Scarsi, R., and Barker, P. S. (1944). Amer. Heart J., 27, 19. 